\title{
In silico analysis reveals a shared immune signature in CASP8-mutated carcinomas with varying correlations to prognosis
}

\author{
Yashoda Ghanekar Corresp., 1 , Subhashini Sadasivam ${ }^{\text {Corresp. 1,2 }}$ \\ 1 DeepSeeq Bioinformatics, Bangalore, Karnataka, India \\ 2 Institute for Stem Cell Biology and Regenerative Medicine, Bangalore, Karnataka, India \\ Corresponding Authors: Yashoda Ghanekar, Subhashini Sadasivam \\ Email address: yashoda.ghanekar@gmail.com, contact@deepseeq.com
}

Background. Sequencing studies across multiple cancers continue to reveal mutations and genes involved in the pathobiology of these cancers. Exome sequencing of oral cancers, a subset of Head and Neck Squamous cell Carcinomas (HNSCs) common among tobacco-chewing populations, revealed that $\sim 34 \%$ of the affected patients harbor mutations in the CASP8 gene. Uterine Corpus Endometrial Carcinoma (UCEC) is another cancer where $\sim 10 \%$ cases harbor CASP8 mutations. Caspase- 8 , the protease encoded by CASP8 gene, plays a dual role in programmed cell death, which in turn has an important role in tumor cell death and drug resistance. CASP8 is a protease required for the extrinsic pathway of apoptosis and is also a negative regulator of necroptosis. Using multiple tools such as differential gene expression, gene set enrichment, gene ontology, in silico immune cell estimates, and survival analyses to mine data in The Cancer Genome Atlas, we compared the molecular features and survival of these carcinomas with and without CASP8 mutations.

Results. Differential gene expression followed by gene set enrichment analysis showed that HNSCS with CASP8 mutations displayed a prominent signature of genes involved in immune response and inflammation. Analysis of abundance estimates of immune cells in these tumors further revealed that mutant-CASP8 HNSCS were rich in immune cell infiltrates. However, in contrast to Human Papilloma Virus-positive HNSCs that also exhibit high immune cell infiltration, which in turn is correlated with better overall survival, HNSC patients with mutant-CASP8 tumors did not display any survival advantage. Similar analyses of UCECs revealed that while UCECs with CASP8 mutations also displayed an immune signature, they had better overall survival, in contrast to the HNSC scenario. There was also a significant upregulation of neutrophils ( $p$-value $=0.0001638$ ) as well as high levels of IL33 mRNA ( $p$-value $=7.63747 \mathrm{E}$ 08 ) in mutant-CASP8 HNSCs, which were not observed in mutant-CASP8 UCECs.

Conclusions. These results suggested that carcinomas with mutant CASP8 have broadly similar immune signatures albeit with different effects on survival. We hypothesize that subtle tissue-dependent differences could influence survival by modifying the micro-environment of mutant-CASP8 carcinomas. High neutrophil numbers, a well-known negative prognosticator in HNSCs, and/or high IL33 levels may be some of the factors affecting survival of mutant-CASP8 cases. 


\section{In Silico Analysis Reveals a Shared Immune Signature in CASP8-Mutated Carcinomas} with Varying Correlations to Prognosis

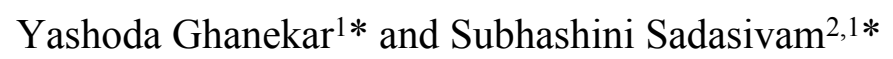

Subhashini Sadasivam

DeepSeeq Bioinformatics, Ananthapura, Yelahanka, Bangalore 560064, India Email address: contact@deepseeq.com, subhashini.sadasivam@gmail.com

\section{Abstract}

Background. Sequencing studies across multiple cancers continue to reveal mutations and genes involved in the pathobiology of these cancers. Exome sequencing of oral cancers, a subset of Head and Neck Squamous cell Carcinomas (HNSCs) common among tobacco-chewing populations, revealed that $\sim 34 \%$ of the affected patients harbor mutations in the CASP 8 gene. Uterine Corpus Endometrial Carcinoma (UCEC) is another cancer where $\sim 10 \%$ cases harbor CASP8 mutations. Caspase-8, the protease encoded by CASP8 gene, plays a dual role in programmed cell death, which in turn has an important role in tumor cell death and drug resistance. CASP8 is a protease required for the extrinsic pathway of apoptosis and is also a negative regulator of necroptosis. Using multiple tools such as differential gene expression, gene set enrichment, gene ontology, in silico immune cell estimates, and survival analyses to mine data in The Cancer Genome Atlas, we compared the molecular features and survival of these carcinomas with and without CASP 8 mutations.

Results. Differential gene expression followed by gene set enrichment analysis showed that HNSCs with CASP8 mutations displayed a prominent signature of genes involved in immune response and inflammation. Analysis of abundance estimates of immune cells in these tumors further revealed that mutant-CASP 8 HNSCs were rich in immune cell infiltrates. However, in contrast to Human Papilloma Virus-positive HNSCs that also exhibit high immune cell infiltration, which in turn is correlated with better overall survival, HNSC patients with mutantCASP8 tumors did not display any survival advantage. Similar analyses of UCECs revealed that while UCECs with CASP8 mutations also displayed an immune signature, they had better overall 
41 survival, in contrast to the HNSC scenario. There was also a significant up-regulation of

42 neutrophils ( $\mathrm{p}$-value $=0.0001638$ ) as well as high levels of IL33 mRNA (p-value $=7.63747 \mathrm{E}-08$ )

43 in mutant-CASP8 HNSCs, which were not observed in mutant-CASP8 UCECs.

44 Conclusions. These results suggested that carcinomas with mutant $C A S P 8$ have broadly similar 45 immune signatures albeit with different effects on survival. We hypothesize that subtle tissue-

46 dependent differences could influence survival by modifying the micro-environment of mutant-

47 CASP8 carcinomas. High neutrophil numbers, a well-known negative prognosticator in HNSCs,

48

49

50

51

52

53

54

55

56

57

58

59

60

61

62

63

64

65

66

67

68

69

70

71

72

73

74

75

76

77

78

79 and/or high IL33 levels may be some of the factors affecting survival of mutant-CASP 8 cases.

\section{Introduction}

Exome sequencing, RNA-sequencing, and copy number variation analysis of different cancers have revealed a cornucopia of disease-relevant mutations and altered pathways ( 1 ). The identified genes included those with broad relevance across different cancers, as well as those relevant in one or few cancer types. The next phase will involve parsing this voluminous data to generate ideas and hypotheses with the potential for clinical impact, and then testing them experimentally.

We are particularly interested in the heterogeneous group of Head and Neck Squamous cell Carcinomas (HNSCs) as these account for a large number of mortalities each year in the Indian subcontinent $(2,3)$. Multiple exome sequencing studies have revealed the landscape of recurrent somatic mutations in HNSCs and its prevalent subtype of Oral Squamous Cell Carcinomas (OSCCs) (4-8). While TP53 was the most significant recurrently mutated gene in this cancer type, several other genes such as CASP8, FATI, and NOTCH1 were also unearthed as significantly recurrently mutated by these large-scale sequencing studies. Barring TP53, the roles of these genes in oral epithelium homeostasis, and how this is altered owing to their mutation in cancer remain to be fully elucidated (9). In this study, we chose to focus on the CASP8 gene, which is mutated in $\sim 10 \%$ of all HNSC cases, and more specifically in $34 \%$ of cases with OSCC of the gingiva-buccal sulcus (OSCC-GB), the subtype that accounts for the majority of HNSC cases in the Indian subcontinent $(4,7,8)$. The types of mutations in CASP8 reported in these HNSC cases included loss of function due to frameshift, nonsense mutation or splice mutation as well as missense and deletion mutations.

Apart from HNSC, Uterine Corpus Endometrial Carcinoma (UCECs) carried the most numbers of mutations in the CASP8 gene, as was observed upon searching the Genomic Data Commons (10). We found that $C A S P 8$ was recurrently mutated in about $10 \%$ of UCEC cases. Here again, the role of CASP 8 in endometrial tissue homeostasis, and how this is altered owing to its mutation in UCEC remains unclear. CASP 8 was also mutated in other cancer types, however, the numbers of such tumors are too low for meaningful analyses. Thus, using the sequencing data on 528 head and neck, and 560 uterine corpus endometrial carcinoma tumors available in The Cancer Genome Atlas (TCGA) $(11,12)$, we sought to identify distinctive features of mutantCASP8 tumors. 
80 CASP8 regulates two pathways of programmed cell death; it is a key protease required for the

81

82

83

84

85

86

87

88

89

90

91

92

93

94

95

96

97

98

99

100

101

102

103

104

105

106

107

108

109

110

111

112

113

114

115

116

117

118

initiation of the extrinsic apoptotic pathway that is targeted by some drug-resistant tumors, and it is an important negative regulator of necroptosis (13-10). Loss-of-function mutations in CASP8 could lead to reduced apoptosis and promote tumor survival (17). It could also lead to enhanced necroptosis and promote tumor cell death $(15,16)$. Interestingly, it has been proposed that the necroptotic pathway could be utilized to develop anti-cancer treatments for countering cancers with resistance to apoptosis (18). At least four HNSC-associated CASP8 mutations have been reported to inhibit activation of the extrinsic apoptosis pathway suggesting loss-of-function, however necroptosis was not analyzed in this study (19). On the background of these observations, tumors harboring $C A S P 8$ mutations offer a tractable, physiologically relevant opportunity to understand the changes brought about by $C A S P 8$ mutation, how it affects survival, and if $C A S P 8$ or the necroptotic pathway could be a potential drug target.

In this study, we describe the comparison of RNA-sequencing (RNA-seq) data from head and neck squamous cell carcinoma, and later from uterine corpus endometrial carcinoma, that are mutant or wild type for $C A S P 8$. We report distinctive molecular features of mutant-CASP 8 HNSCs and UCECs that this comparison revealed. In addition, we describe results obtained by correlating these features to overall survival in the affected patients.

\section{Materials and Methods}

\section{Differential Gene Expression Analysis of wild-type-CASP8 and mutant-CASP8 cases}

Data for 528 head and neck squamous cell carcinoma (HNSC) cases available at The Cancer Genome Atlas (TCGA) were downloaded in May-June 2017 from https://portal.gdc.cancer.gov/. Clinical data files, Mutation Annotation Format (MAF) files, and mRNA quantification files such as HT-Seq files (files with number of reads aligning to each protein-coding gene) and FPKM-UQ files (files with number of fragments aligning per kilobase of transcript per million mapped reads normalized to upper quartile) were downloaded. The HPV status of HNSC cases at TCGA has been reported earlier (20), and these data were used to assign HPV-positive and HPVnegative cases.

Cases with and without CASP8 mutation were selected as shown in Fig. 1. CASP8 mutations in HNSC cases were identified using the Mutation Annotation Format (MAF) files available at TCGA. The workflow for somatic mutation calling at TCGA uses four different pipelines:

SomaticSniper, MuSE, MuTect2, and VarScan2. The variants called by these four pipelines are further annotated to infer the biological context of each variant using Variant Effect Predictor (VEP). VEP predicts the effect of variants based on its location and information from databases such as GENCODE, sift, ESP, polyphen, dbSNP, Ensembl genebuild, Ensembl regbuild, HGMD and ClinVar. This annotation results in a list of variants with three predicted effects; high impact variants arising from frame-shift or nonsense mutations, variants with moderate impact which include missense mutations and low impact which include variants with synonymous mutations. 
119 The information regarding the impact of mutations was available in the MAF file from each

120 somatic mutation calling pipeline employed by TCGA.

$12155 \mathrm{HNSC}$ cases with non-synonymous CASP8 mutations were identified from MAF files.

122 Notably, the majority ( $80 \%$ ) of the identified CASP 8 mutations were predicted by more than one

123 somatic mutation calling pipeline, and all had either high or moderate impact on function. All

124 cases with CASP 8 mutation were HPV-negative and were found in tumors at specific sites in oral

125 cavity. Therefore, HNSC cases that were from these same subsites and were HPV-negative were

126 used as wild type control. A total of 424 HNSC cases of which 369 had wild-type-CASP8

127 (CASP8-WT) and 55 had mutant-CASP8 (CASP8-MT) were thus selected (Table S1). Among

128 selected cases, RNA-seq data was available for 354 cases with wild-type-CASP 8 and 53 cases

129 with mutant-CASP8.

130 Transcripts that were differentially expressed in CASP8-MT as compared to CASP8-WT cases

131 were identified using edgeR (21). edgeR uses raw read counts as input, which were obtained

132 from HT-Seq files. The analysis was performed using quantile-adjusted conditional maximum

133 likelihood (qCML) method without any filters. All transcripts with FDR $<0.001$ and showing a

134 fold-change of at least 2.5 -fold ( $\operatorname{logFC}$ of 1.3 ) were deemed to be significantly differentially

135 expressed.

136 Similarly, clinical data and HT-Seq files for 560 uterine corpus endometrial carcinoma (UCEC)

137 cases available at TCGA were downloaded in February 2018. CASP 8 mutations of high or

138 moderate impact were present in 56 UCEC cases. Cases without $C A S P 8$ mutations were used as

139 wild type control. RNA-seq data was available for 476 CASP8-WT tumors and 56 CASP8-MT

140 tumors. Transcripts that were differentially expressed in CASP8-MT as compared to CASP8-WT

141 cases were identified using edgeR as described for differential gene expression analysis of

142 HNSC.

143

144 Gene Ontology and Gene Set Enrichment Analysis (GSEA)

145 Enrichment analysis was performed at http://geneontology.org/to identify biological processes

146 overrepresented among transcripts that were differentially expressed between CASP8-WT and

147 CASP8-MT HNSCs (22). Genes that passed the following criteria: (a) FDR $<0.001$ (b)

$148 \mathrm{FDR}<0.001$ and $\log 2 \mathrm{FC}<-1.3$, (c) $\mathrm{FDR}<0.001$ and $\log 2 \mathrm{FC}>1.3$, (d) $\mathrm{b}$ and $\mathrm{c}$ merged, were used to

149 create input gene sets for gene ontology analysis performed using PANTHER version 13.1

150 (release 2018-02-03). The Binomial test was used to determine statistical significance and the

151 Bonferroni correction for multiple testing was applied.

152 GSEA was performed using a pre-ranked gene list and hallmark gene sets available at the

153 Molecular Signature Database (23). The hallmark gene sets use either HGNC or entrez gene ids

154 as the gene identifier. Out of the 60,483 transcripts analyzed by edgeR, HGNC gene symbols

155 could be assigned to 36,095. $\log \mathrm{FC}$ values for these 36,095 transcripts from the edgeR output

156 from HNSC and UCEC differential gene expression analyses were used to generate the pre-

157 ranked gene list. Gene sets with FDR $<25 \%$ and with distinct enrichment at the beginning or end

158 of the ranked list (as observed in enrichment plots) were taken to be significantly enriched gene 
159

160

161

162

163

164

165

166

167

168

169

170

171

172

173

174

175

176

177

178

179

180

181

182

183

184

185

186

187

188

189

190

191

192

193

194

195

196

197

198

sets. To perform GSEA with genes that were up-regulated in the skin of mice lacking functional Caspase-8, the top 100 up-regulated genes that were reported were selected (24). Of these 100 genes, 80 genes had corresponding human orthologs (CASP8-KOSET) as identified using tools available at http://www.informatics.jax.org/. GSEA was then performed using the pre-ranked gene list and the CASP8-KOSET.

\section{Immune Cell Infiltration in HNSC and UCEC cases}

The abundance estimates of six immune cell types; $\mathrm{B}$ cells, $\mathrm{CD} 4^{+} \mathrm{T}$ cells, $\mathrm{CD} 8^{+} \mathrm{T}$ cells, Neutrophils, Macrophages, and Dendritic cells in TCGA cases was downloaded from Tumor IMmune Estimation Resource (TIMER) at https://cistrome.shinyapps.io/timer/ (25). This data was available for 353 CASP8-WT and 51 CASP8-MT cases from HNSC. The comparison of immune cell infiltration levels across CASP8-WT, CASP8-MT, and HPV-positive cases was performed using a two-sided Wilcoxon rank test and the graphs were plotted using R. Similar analysis was performed for all CASP8-MT and CASP8-WT cases from UCEC.

\section{Survival Analysis}

Survival analysis was performed to investigate the difference in the survival of CASP8-WT and CASP8-MT patients from HNSC and UCEC. Survival analysis was also performed to investigate the effect of factors such as expression levels of certain genes and immune cell infiltration on survival. The expression levels of genes of interest were obtained from FPKM-UQ files from TCGA and the data for distribution of immune cell infiltration was obtained from TIMER. Kaplan-Meier curves for $C A S P 8$-WT and CASP8-MT cases were plotted using the Survival and Survminer packages in $\mathrm{R}$ and the plots were compared using the log-rank test (26).

To investigate the effect of genes of interest (such as those from gene sets enriched in GSEA or genes involved in necroptosis) and immune cell infiltration levels on survival, multivariate Cox proportional hazards test was performed for HNSC cases. In addition, Cutoff Finder tool available at http://molpath.charite.de/cutoff/ was used to investigate the influence of a single continuous variable on survival (27). In the Cutoff Finder tool, the cutoff for dichotomization of a continuous variable was determined as the point with the most significant split by log-rank test, using coxph and survfit functions from the R package survival. Survival analysis of either CASP8-WT or CASP8-MT cases was performed using this method by dichotomizing gene expression or immune cell infiltration levels.

\section{Results}

\section{Genes involved in immune response are up-regulated in mutant-CASP8 HNSCs}

To investigate the significance of CASP 8 mutations in head and neck squamous cell carcinoma (HNSC), we performed differential gene expression analysis using RNA-seq data from HNSC cases with and without $C A S P 8$ mutations. As reported previously, HNSCs carrying CASP8 mutations occurred predominantly in sites within the oral cavity such as the cheek mucosa, floor of mouth, tongue, larynx, and overlapping sites of the lip, oral cavity, and pharynx (Table S1). In 
199

200

201

202

203

204

205

206

207

208

209

210

211

212

213

214

215

216

217

218

219

220

221

222

223

224

225

226

227

228

229

230

231

232

233

234

235

236

237

238

addition, since HPV-positive (Human Papillomavirus-positive) HNSCs constitute a molecularly distinct subtype; we examined the HPV status of the 55 mutant-CASP8 HNSCs using data from Chakravarthy et al (20). Based on this reported data, all 55 mutant-CASP8 HNSCs were found to be HPV-negative. Since all the HNSC cases carrying CASP8 mutations were HPV-negative, and were from specific sites within the oral cavity, HNSCs carrying wild-type-CASP 8 that were HPV-negative and also from these same sites were selected as controls for all subsequent analyses. A total of 424 HNSC cases of which 369 had wild-type-CASP8 (CASP8-WT) and 55 had mutant-CASP8 (CASP8-MT) were thus selected (Fig. 1, see also Table S1). Of these, RNAseq data was available for $354 C A S P 8$-WT and 53 CASP8-MT cases.

Raw sequencing reads from CASP8-WT and CASP8-MT cases, obtained from HT-Seq files, were subjected to edgeR analysis for differential gene expression (Table S2). At FDR $<0.001$, 186 genes were up-regulated in $C A S P 8$-MT with $\log 2 \mathrm{FC}>1.3$ while 1139 genes were downregulated in $C A S P 8$-MT with $\log 2 \mathrm{FC}<-1.3$ (Fig. 2A). There was also a statistically significant 1.3-fold increase in the expression level of the CASP8 gene perhaps to overcome the loss of function (Table S2, gene ESNG00000064012 in HNSC edgeR output).

To identify biological processes specifically enriched in the CASP8-WT or CASP8-MT cases, enrichment analysis was performed with the differentially expressed genes using tools available at the Gene Ontology (GO) Consortium. As seen in Fig. 2A, distinct processes were enriched in the CASP8-WT and CASP8-MT cases. For example, genes involved in the regulation of immune response ( $\mathrm{p}$-value $=3.30 \mathrm{E}-02$ ) were enriched in CASP8-MT HNSCs while genes with roles in synaptic transmission ( $\mathrm{p}$-value $=2.85 \mathrm{E}-07)$, synaptic vesicle exocytosis $(\mathrm{p}$-value $=3.90 \mathrm{E}-03$ ), and muscle contraction ( $\mathrm{p}$-value $=2.29 \mathrm{E}-03$ ) were the top three biological processes enriched in CASP8-WT HNSCs. Please refer to Table S3 for the full list.

We further analyzed the differential gene expression data using the Gene Set Enrichment Analysis (GSEA) tool. After generating a pre-ranked gene list based on $\operatorname{logFC}$ values from the edgeR analysis, we queried this list in the GSEA software using hallmark gene sets available at the Molecular Signatures Database. Several gene sets were enriched in upregulated or downregulated genes in CASP8-MT cases at FDR $<25 \%$. Particularly, gene sets involved in immune regulation such as allograft rejection, complement, inflammatory response, interferon- $\alpha$ response, and interferon- $\gamma$ response, were specifically enriched in the CASP8-MT HNSCs, in sync with the GO results (Fig. 2B and Table S4). The hallmark gene sets enriched in CASP8-WT HNSCs were epithelial-mesenchymal transition (EMT), myogenesis, and the KRAS pathway (Fig. 2B and Table S4).

\section{Gene expression in the skins of epidermal Caspase- 8 knockout mice mirrors the expression pattern of mutant-CASP8 HNSCs}

Expression of an enzymatically inactive Caspase- 8 mutant or the deletion of wild-type Caspase8 in the mouse epidermis leads to chronic skin inflammation $(24,28)$. A microarray analysis performed by Kovalenko et al. to identify genes specifically up-regulated in the skin epidermis of Casp- $8^{F /} \mathrm{K} 5$-Cre (relative to Casp- $8^{F /+} \mathrm{K} 5$-Cre epidermis) mice revealed increased expression 
239

240

241

242

243

244

245

246

247

248

249

250

251

252

253

254

255

256

257

258

259

260

261

262

263

264

265

266

267

268

269

270

271

272

273

274

275

276

277

278

of several immune-regulatory and inflammatory genes including several cytokines. Using the human orthologs of these up-regulated genes (Table S5), we again queried the pre-ranked gene list with the GSEA tool. As seen in Fig. $2 \mathrm{C}$, genes highly expressed in the Casp- $8^{F /} \mathrm{K} 5$-Cre mouse skins were also significantly enriched in CASP8-MT HNSCs (as opposed to their wildtype counterparts), indicating that the inactivation of CASP 8 leads to the up-regulation of a similar set of genes in both mouse and human epidermal tissues.

\section{Enrichment of immune response gene sets correlates with increased infiltration of specific} immune cell types in mutant-CASP8 HNSCs

Gene sets involved in immune response were specifically enriched in CASP8-MT HNSCs. HPVpositive HNSCs, a subset of HNSCs, also display high immune cell infiltration, as compared to HPV-negative HNSCs $(20,29-31)$. We investigated if the enrichment of immune response genes in CASP8-MT HNSCs was correlated with increased infiltration of immune cells, and if it was comparable to the immune cell infiltration levels in HPV-positive HNSCs. Immune cell infiltration levels in three subsets of HNSCs; CASP8-WT, CASP8-MT (both HPV-negative), and HPV-positive (which is CASP8-WT), were compared using the Wilcoxon test; the comparisons were: (1) CASP8-WT and CASP8-MT (2) CASP8-WT and HPV-positive CASP8-WT, and (3) $C A S P 8$-MT and HPV-positive CASP8-WT. We checked if there was a difference in the numbers/types of immune cell infiltrates between these three subsets of HNSCs using the data available at Tumor IMmune Estimation Resource (TIMER) (25), a comprehensive resource for immune cell infiltration of TCGA tumors.

Consistent with the GSEA results, CASP8-MT cases showed significantly higher infiltration of $\mathrm{CD}^{+} \mathrm{T}$ cells, neutrophils, and dendritic cells as compared to CASP8-WT cases (pvalues $<0.0005)$, suggesting that the immune response to the tumor in WT and MT cases was different (Fig. 3A). Also, in agreement with previous reports, HPV-positive HNSCs had significantly higher infiltration of all immune cell types as compared to the CASP8-WT HNSCs. A comparison of immune cell infiltration levels in HPV-positive and CASP8-MT HNSCs showed that the extent of infiltration of $\mathrm{CD}^{+} \mathrm{T}$ cells, neutrophils, and dendritic cells (Fig. 3A) in these two subsets was also similar. However, HPV-positive HNSCs had higher infiltration of $\mathrm{CD}^{+} \mathrm{T}$ cells and B cells, compared to the other two subsets (Fig. 3B).

\section{The "immune signature" of mutant-CASP8 HNSCs does not correlate to improved overall survival}

High levels of immune cell infiltration in HPV-positive cases correlates with better survival in HPV-positive HNSC cases $(30,31)$. To investigate if a similar effect could be observed in the survival of HNSC patients with and without CASP8 mutation, Kaplan-Meier analysis was performed on the CASP8-WT and CASP8-MT cases (filtered as per the schema in Fig. 1). There was no significant difference in the survival of patients with and without CASP8 mutations (pvalue $=0.16$, Fig. $4 \mathrm{~A}$ ), indicating that high levels of immune cell infiltration may not necessarily corelate with better survival in HNSCs. 
279 The effect of genes from pathways enriched either in CASP8-WT or CASP8-MT tumors (listed 280 in Table S4) on survival was then investigated using the Cox proportional hazards model. Four

281

282

283

284

285

286

287

288

289

290

291

292

293

294

295

296

297

298

299

300

301

302

303

304

305

306

307

308

309

310

311

312

313

314

315

316

317

318 genes from pathways enriched in CASP8-MT HNSCs; PRF1, CXCR6, CD3D, and GZMB, reduced the hazard ratio significantly in $C A S P 8$-WT cases, at $\mathrm{p}<0.05$ (Table S6). We also performed the survival analysis using Cutoff Finder to investigate the effect of the expression of individual genes on the survival of CASP8-WT and CASP8-MT cases. Increased expression of all these four genes was associated with higher overall survival in $C A S P 8$-WT (at $\mathrm{p}<0.05$ ). In contrast, in CASP8-MT cases, such association was seen only with GZMB expression levels (Fig. $4 \mathrm{~B}$ and Fig. S1). Similarly, higher $\mathrm{CD} 8^{+} \mathrm{T}$ cell estimates (from TIMER) was also significantly associated with better survival in CASP8-WT but not in CASP8-MT HNSCs (Fig. 4B).

Since CASP8 is a negative regulator of the necroptotic pathway [11,12], we also investigated the effect of expression levels of genes involved in necroptosis on survival. Higher expression of RIPK1, RIPK3, and MLKL was associated with higher overall survival in CASP8-WT but not in CASP8-MT cases (Fig. S2). Additional factors that influenced survival are shown in Table S6.

\section{Mutant-CASP8 UCECs exhibit an immune signature similar to mutant-CASP8 HNSCs}

We then investigated if this effect seen in CASP8-MT HNSCs was broadly applicable across other cancers carrying $C A S P 8$ mutations. On searching the Genomic Data Commons, we found that $C A S P 8$ was recurrently mutated in about $10 \%$ of (UCEC) cases. From a total of 560 UCEC cases, RNA-seq data was available for 476 CASP8-WT and 56 CASP8-MT cases. HTSeq files containing raw sequencing reads from these two groups were subjected to edgeR analysis for differential gene expression and further analyzed using the GSEA tool. Several gene sets involved in immune regulation were specifically enriched in the CASP8-MT UCECs. Notably, categories such as allograft rejection, interferon- $\alpha$ response, and interferon- $\gamma$ response, were enriched in the CASP8-MT UCECs similar to the HNSC results (Fig. 5A and Fig. 2B). The genes that contributed to core enrichment in these gene sets in CASP8-MT UCECs also contributed to core enrichment of the same gene sets in CASP8-MT HNSCs, indicating that similar immune response genes were upregulated in the two carcinomas (Table S7). However, unlike HNSCs, the gene set for inflammatory response did not show any enrichment in CASP8MT UCECs. CASP8-MT UCECs were additionally enriched for genes involved in apoptosis. Notably, this was not observed in the CASP8-MT HNSCs (Fig. 2B and Fig. 5A).

\section{High levels of IL33 and neutrophil infiltration are observed in mutant-CASP8 HNSCs but not in mutant-CASP8 UCECs}

Using TIMER, we then checked the levels of infiltrating immune cells in the CASP8-WT and CASP8-MT UCECs. Consistent with the GSEA results, CASP8-MT UCEC cases showed significantly higher infiltration of $\mathrm{CD} 8^{+} \mathrm{T}$ cells and dendritic cells as compared to CASP8-WT cases (p-values $<0.005$ ). However, in contrast to the HNSC data, the levels of neutrophils were not significantly higher in the CASP8-MT UCEC group (Fig. 5B, see also Fig. 3A). We then investigated if differences in the levels of neutrophil-active chemokines could potentially explain 
319 this observation (32). From the edgeR differential expression data comparing the CASP8-MT 320 and CASP8-WT groups in HNSC and UCEC, we obtained the fold change values and statistical 321 significance of different chemokines known to attract neutrophils (Table S8). Interestingly, the 322 cytokine IL33 was significantly up regulated (1.8 fold, FDR $<0.001)$ in CASP8-MT HNSCs but 323 not in CASP8-MT UCECs.

324 Next, we performed Kaplan-Meier analysis on CASP8-WT and CASP8-MT UCEC cases. In 325 contrast to the HNSC survival data, there was a difference in the survival of UCEC cases with 326 and without $C A S P 8$ mutations, with cases harboring CASP8 mutations reporting better overall

327

328

329

330

331

332

333

334

335

336

337

338

339

340

341

342

343

344

345

346

347

348

349

350

351

352

353

354

355

356

357

358 survival (p-value $=0.019$, Fig. $5 C$ ).

\section{Discussion}

Here, we report a distinct class of carcinomas that have mutated CASP8. Using bioinformatics approaches to mine the TCGA data, we identified high expression of immune response-related genes (listed in Table S7) combined with high infiltration of $\mathrm{CD} 8^{+} \mathrm{T}$ cells and dendritic cells as a prominent shared immune signature in CASP8-MT carcinomas. In the first part of our analyses, we investigated the implications of the enrichment of this immune signature across different HNSC subtypes. Subsequently, in the second part, we investigated the correlation between immune signature and survival in two carcinomas having a significant number of cases with CASP8 mutations, HNSC and UCEC. Our analyses showed that despite similarities in the enrichment of gene sets, these carcinomas exhibited varying correlations of immune signature with survival. Our studies indicated that tissue-specific differences, such as the levels of infiltrating neutrophils and the cytokine IL33, could be responsible for the varying correlation of immune signature with survival.

Multiple studies have reported that HPV-positive HNSCs display a strong immune signature and high infiltration of immune cells that correlates with better survival $(30,31)$. In contrast, our studies show that the enrichment of immune response genes and infiltration of immune cells seen in CASP8-MT HNSCs does not appear to correlate with improved prognosis. In fact, CASP8 mutation leads to the loss of a survival advantage that is observed in HNSC patients with wildtype $C A S P 8$ tumors under certain conditions. For example, higher expression levels of genes such as PRF1, CD3D, and CXCR6 are associated with better survival in CASP8-WT but not in $C A S P 8-M T$. It is possible that the higher expression of these genes results in higher extent of apoptosis leading to survival advantage. This perhaps does not take place in CASP8-MT, leading to the loss of survival advantage from higher expression of these genes. These results argue that a tumor microenvironment with high infiltration of immune cells does not necessarily provide a survival benefit in HNSCs. However, it is important to note that while the sample numbers of CASP8-MT cases are significant $(\mathrm{n}=55)$, it is lesser than the number of CASP8-WT cases $(\mathrm{n}=369)$. This may influence $\mathrm{p}$-values, and it will be necessary to confirm these findings with greater numbers of CASP8-MT cases once more data becomes available.

We can think of at least two potential scenarios to explain the increased immune cell infiltration observed in CASP8-MT tumors. (a) Unregulated inflammatory and wound healing response: As 
359

360

361

362

363

364

365

366

367

368

369

370

371

372

373

374

375

376

377

378

379

380

381

382

383

384

385

386

387

388

389

390

391

392

393

394

395

396

397

398

mentioned earlier, loss of Caspase-8 in the mouse epidermis leads to chronic inflammation (24). The infiltration of immune cells in mucosa lacking CASP 8 accompanied by the enrichment of immune-associated gene sets is highly reminiscent of this phenotype. It has also been proposed that the loss of Caspase- 8 in the mouse skin epidermis simulates a wound healing response (28). Both scenarios involve a gamut of immune cell types and secreted cytokine factors, leading to immune cell infiltration. It should however be noted that although similar gene sets are enriched in mouse skins lacking Caspase- 8 and in CASP8-MT tumors, the types of immune cell infiltrates in the two are different. (b) Necroptosis: More recently, several studies have revealed a role for Caspase- 8 as an inhibitor of necroptosis, a highly pro-inflammatory mode of cell death $(13,14)$. In intestinal epithelia, the loss of Caspase- 8 promoted necroptosis through the activation of RIP kinases and MLKL $(15,16)$. A similar scenario could be occurring in CASP8-MT tumors leading to the expression of pro-inflammatory genes and the infiltration of immune cells.

Why doesn't the increased number of immune cells translate into improved prognosis in CASP8MT HNSC tumors? Since CASP8 is an important mediator of the extrinsic apoptotic pathway, CASP8-MT tumors may have greater resistance to Fas- or DR5- mediated cell death pathways, which are typically employed by $\mathrm{CD} 8^{+} \mathrm{T}$ cells and Natural Killer cells to target infected/tumor cells $(19,33)$. The survival analysis carried out in this study showed that CASP8-WT HNSC patients with higher expression of genes involved in T-cell mediated cytotoxicity had better survival. Importantly, this advantage was not seen in CASP8-MT patients.

Several studies have reported that high neutrophil numbers and an elevated neutrophil/lymphocyte ratio portended poorer prognosis in $\operatorname{OSCC}(34,35)$. Thus, it is possible that elevated levels of neutrophil infiltration seen in CASP8-MT HNSC cases could be one of several events contributing to the poorer prognosis of CASP8-MT HNSCs. IL33, a cytokine and an alarmin linked to necroptosis may represent a possible mechanism for neutrophil recruitment in these cases $(36,37)$. High IL33 levels are also associated with poor prognosis in HNSCs (38). In addition, the pro-inflammatory environment generated during necroptosis may hold other advantages for the survival of CASP8-MT HNSCs. Necroptosis, IL33 levels, and neutrophil infiltration together or through independent mechanisms could be leading to a pro-tumor environment. Thus, promoting necroptosis may not necessarily translate into better survival for HNSC patients with apoptosis-resistant tumors.

Another reason for the lack of survival advantage in CASP8-MT HNSCs could be the composition of tumor-infiltrating immune cells in these tumors. For instance, HPV-positive tumors had higher levels of $\mathrm{B}$ cells and $\mathrm{CD}^{+} \mathrm{T}$ cells as compared to CASP8-MT tumors. It is likely that in addition to cytotoxic $\mathrm{T}$ cells, $\mathrm{B}$ cells and $\mathrm{CD} 4^{+} \mathrm{T}$ cells are required to mediate an immune response essential for tumor cell death, possibly for tumor antigen presentation or cytokine secretion.

A comparison of CASP8-MT HNSCs and CASP8-MT UCECs highlighted similarities and differences between the two carcinomas. Both CASP8-MT HNSCs and CASP8-MT UCECs showed an enrichment of gene sets involved in immune response such as interferon $\alpha$ response, interferon $\gamma$ response, and allograft rejection. Most of the genes contributing to core enrichment 
399

400

401

402

403

404

405

406

407

408

409

410

411

412

413

414

415

416

417

418

419

420

421

422

423

424

425

426

427

428

429

430

431

432

433

434

435

436

437

438

in these gene sets in UCECs also contributed to core enrichment of these gene sets in HNSCs. Moreover, both these carcinomas showed high infiltration of $\mathrm{CD} 8^{+} \mathrm{T}$ cells and dendritic cells but not $\mathrm{B}$ cells or $\mathrm{CD}^{+} \mathrm{T}$ cells. CASP8 mutation thus led to a similar immune response in both HNSCs and UCECs. This shared immune signature, however, did corelate with a specific survival outcome. Notably, CASP8-MT UCECs showed a significant survival advantage over CASP8-WT UCECs, unlike its HNSC counterpart. While we do not yet know the causal reason(s), the differences per se may be worth noting and could be responsible for this advantage. For instance, in contrast to CASP8-MT HNSCs, the gene set for inflammatory response was not enriched but the gene set for apoptosis was enriched in CASP8-MT UCECs. There was also no increased infiltration of neutrophils or transcriptional upregulation of IL33 in CASP8-MT UCECs. The up-regulation of apoptotic pathways together with the lack of enrichment of an inflammation-associated gene set that is typical of necroptosis perhaps indicates that apoptosis, rather than necroptosis, is the predominant mode of programmed cell death in CASP8-MT UCECs. This lack of inflammation may also be responsible for the lack of neutrophil infiltration in CASP8-MT UCECs since neutrophil chemoattractants, such as IL33, may not be released during apoptosis but is perhaps released during the highly inflammatory process of necroptosis, in turn leading to neutrophil infiltration.

It is also possible that necroptosis is initiated in CASP8-MT UCECs but the accompanying IL33 up-regulation and/or neutrophil infiltration seen in HNSCs does not take place due to tissuespecific differences. Under such conditions, apoptosis and necroptosis together could provide the survival advantage that is observed in CASP8-MT UCECs. Thus, in contrast to HNSCs, Caspase8 pathway can be explored to identify potential drug targets in UCECs.

\section{Conclusions}

In this in silico study, we explore the implications of CASP8 mutations that have been identified across carcinomas through large-scale genomic studies. Our studies show that CASP8-mutated carcinomas display a shared immune signature. However, the consequences of this immune signature vary with CASP8-MT UCECs showing better survival while CASP8-MT HNSC cases do not have any survival advantage. Our analyses further suggest that neutrophil numbers and IL33 levels could be potential factors affecting the survival of mutant-CASP 8 carcinomas. Broadly, our study highlights the need to further investigate the interaction between pathways of programmed cell death, immune response, and survival in carcinomas. Such studies could open a new window for therapeutic intervention in CASP8-mutated carcinomas.

\section{Acknowledgements}

The results shown here are based upon data generated by the TCGA Research Network: http://cancergenome.nih.gov/. We thank patients who donated samples to the TCGA and consented to share the resulting data. We also wish to thank TCGA for the unrestricted access provided to the data used in this work. We would like to thank Amrendra Mishra (Hannover Biomedical Research School), Urvashi Bahadur (Strand Life Sciences), and Colin Jamora 
439 (inStem) for their helpful comments on this manuscript. SS thanks S. Ramaswamy (inStem) for

440 his support.

441

442 References

443 1. J. N. Weinstein et al., The Cancer Genome Atlas Pan-Cancer analysis project. Nat Genet $444 \quad 45,1113-1120$ (2013).

445 2. J. Ferlay et al., Estimates of worldwide burden of cancer in 2008: GLOBOCAN 2008. Int J $446 \quad$ Cancer 127, 2893-2917 (2010).

447 3. P. C. Gupta, C. S. Ray, D. N. Sinha, P. K. Singh, Smokeless tobacco: a major public health

448

449

450

451

452

453

454

455

456

457

458

459

460

461

462

463

464

465

466

467

468

469

470

471

472 problem in the SEA region: a review. Indian J Public Health 55, 199-209 (2011).

4. N. Agrawal et al., Exome sequencing of head and neck squamous cell carcinoma reveals inactivating mutations in NOTCH1. Science 333, 1154-1157 (2011).

5. I. P. T. o. t. I. C. G. Consortium, Mutational landscape of gingivo-buccal oral squamous cell carcinoma reveals new recurrently-mutated genes and molecular subgroups. Nat Commun 4, 2873 (2013).

6. C. R. Pickering et al., Integrative genomic characterization of oral squamous cell carcinoma identifies frequent somatic drivers. Cancer Discov 3, 770-781 (2013).

7. N. Stransky et al., The mutational landscape of head and neck squamous cell carcinoma. Science 333, 1157-1160 (2011).

8. T. F. Hayes et al., Integrative genomic and functional analysis of human oral squamous cell carcinoma cell lines reveals synergistic effects of FAT1 and CASP8 inactivation. Cancer Lett 383, 106-114 (2016).

9. S. M. Rothenberg, L. W. Ellisen, The molecular pathogenesis of head and neck squamous cell carcinoma. J Clin Invest 122, 1951-1957 (2012).

10. R. L. Grossman et al., Toward a Shared Vision for Cancer Genomic Data. N Engl J Med 375, 1109-1112 (2016).

11. C. G. A. Network, Comprehensive genomic characterization of head and neck squamous cell carcinomas. Nature 517, 576-582 (2015).

12. C. Kandoth et al., Integrated genomic characterization of endometrial carcinoma. Nature 497, 67-73 (2013).

13. M. Pasparakis, P. Vandenabeele, Necroptosis and its role in inflammation. Nature 517, 311-320 (2015).

14. R. Feltham, J. E. Vince, K. E. Lawlor, Caspase-8: not so silently deadly. Clin Trans/ Immunology 6, e124 (2017).

473 15. C. Günther et al., Caspase-8 regulates TNF- $\alpha$-induced epithelial necroptosis and terminal

474

475

476

477

478

479 ileitis. Nature 477, 335-339 (2011).

16. R. Weinlich et al., Protective roles for caspase-8 and cFLIP in adult homeostasis. Cell Rep 5, 340-348 (2013).

17. G. S. Salvesen, C. M. Walsh, Functions of caspase 8: the identified and the mysterious. Semin Immunol 26, 246-252 (2014).

480

18. Z. Su, Z. Yang, L. Xie, J. P. DeWitt, Y. Chen, Cancer therapy in the necroptosis era. Cell Death Differ 23, 748-756 (2016). 
481

482

483

484

485

486

487

488

489

490

491

492

493

494

495

496

497

498

499

500

501

502

503

504

505

506

507

508

509

510

511

512

513

514

515

516

517

518

519

520

521

522

523

19. C. Li, A. M. Egloff, M. Sen, J. R. Grandis, D. E. Johnson, Caspase-8 mutations in head and neck cancer confer resistance to death receptor-mediated apoptosis and enhance migration, invasion, and tumor growth. Mol Oncol 8, 1220-1230 (2014).

20. A. Chakravarthy et al., Human Papillomavirus Drives Tumor Development Throughout the Head and Neck: Improved Prognosis Is Associated With an Immune Response Largely Restricted to the Oropharynx. J Clin Oncol 34, 4132-4141 (2016).

21. M. D. Robinson, D. J. McCarthy, G. K. Smyth, edgeR: a Bioconductor package for differential expression analysis of digital gene expression data. Bioinformatics 26, 139140 (2010).

22. The Gene Ontology Consortium, Expansion of the Gene Ontology knowledgebase and resources. Nucleic Acids Res 45, D331-D338 (2017).

23. A. Subramanian et al., Gene set enrichment analysis: a knowledge-based approach for interpreting genome-wide expression profiles. Proc Natl Acad Sci U S A 102, 15545-15550 (2005).

24. A. Kovalenko et al., Caspase-8 deficiency in epidermal keratinocytes triggers an inflammatory skin disease. J Exp Med 206, 2161-2177 (2009).

25. T. Li et al., TIMER: A Web Server for Comprehensive Analysis of Tumor-Infiltrating Immune Cells. Cancer Res 77, e108-e110 (2017).

26. T. M. Therneau. ( https://CRAN.R-project.org/package=survival, 2015).

27. J. Budczies et al., Cutoff Finder: a comprehensive and straightforward Web application enabling rapid biomarker cutoff optimization. PLoS One 7, e51862 (2012).

28. P. Lee et al., Dynamic expression of epidermal caspase 8 simulates a wound healing response. Nature 458, 519-523 (2009).

29. R. Mandal et al., The head and neck cancer immune landscape and its immunotherapeutic implications. JCl Insight 1, e89829 (2016).

30. N. Nguyen et al., Tumor infiltrating lymphocytes and survival in patients with head and neck squamous cell carcinoma. Head Neck 38, 1074-1084 (2016).

31. S. Russell et al., Immune cell infiltration patterns and survival in head and neck squamous cell carcinoma. Head Neck Oncol 5, 24 (2013).

32. C. D. Sadik, N. D. Kim, A. D. Luster, Neutrophils cascading their way to inflammation. Trends Immunol 32, 452-460 (2011).

33. M. S. Rooney, S. A. Shukla, C. J. Wu, G. Getz, N. Hacohen, Molecular and genetic properties of tumors associated with local immune cytolytic activity. Cell 160, 48-61 (2015).

34. R. Mahalakshmi et al., Neutrophil-to-lymphocyte ratio: A surrogate

marker for prognosis of oral squamous cell carcinoma. Indian Journal of Medical and Paediatric Oncology 39, 8-12 (2018).

35. J. E. Glogauer, C. X. Sun, G. Bradley, M. A. Magalhaes, Neutrophils Increase Oral Squamous Cell Carcinoma Invasion through an Invadopodia-Dependent Pathway. Cancer Immunol Res 3, 1218-1226 (2015).

36. J. C. Alves-Filho et al., Interleukin-33 attenuates sepsis by enhancing neutrophil influx to the site of infection. Nat Med 16, 708-712 (2010).

37. A. J. Hueber et al., IL-33 induces skin inflammation with mast cell and neutrophil activation. Eur J Immunol 41, 2229-2237 (2011). 
524 38. S. F. Chen et al., The paracrine effect of cancer-associated fibroblast-induced interleukin52533 regulates the invasiveness of head and neck squamous cell carcinoma. J Pathol 231, 526 180-189 (2013).

527 


\section{Figure 1}

A flowchart indicating the sequence of processes used to select the HNSC cases used in this study.

HNSC cases with CASP8 mutation were identified using MAF files from TCGA. Out of 528 HNSC cases available at TCGA, 55 cases had mutations in CASP8. All cases with CASP8 mutation were HPV-negative. Hence, HPV-negative wild-type cases were considered for use as control. In addition, as CASP8-MT cases occurred in specific subsites in oral cavity (Supplementary Table 1), CASP8-WT cases from these same subsites were selected as control. Thus, 369 HNSC cases with wild-type-CASP8 were selected as control. Gene expression data was available for 53 cases with CASP8 mutations and 354 cases with wild type CASP8. Data from HT-Seq files of selected cases with CASP8 mutation and corresponding wild-type control cases was analyzed using edgeR to identify genes that were differentially expressed in CASP8-MT HNSCs as compared to CASP8-WT. DEGs: Differentially Expressed Genes, FDR: False Discovery Rate. 
Figure 1

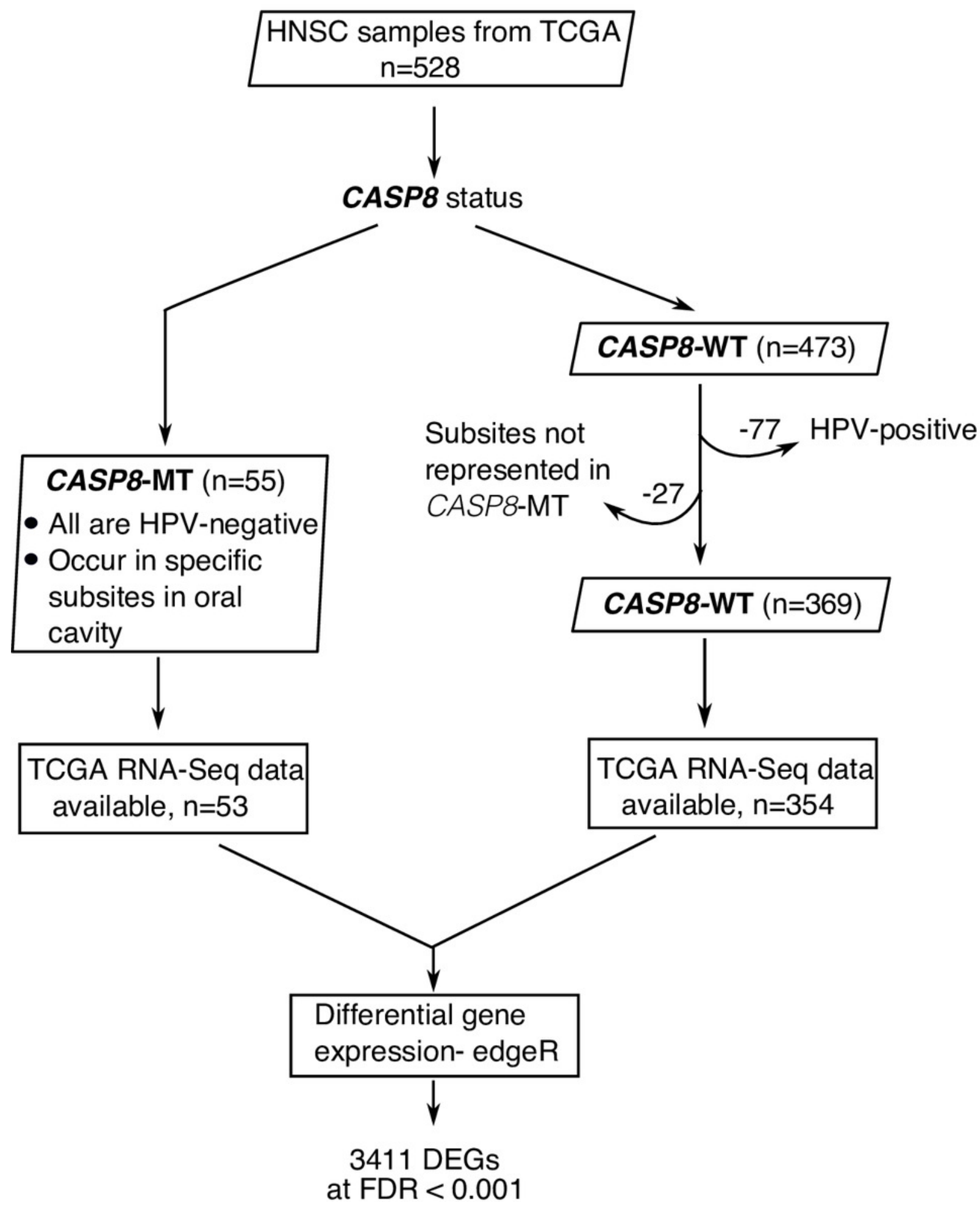




\section{Figure 2}

Gene enrichment analyses reveal a prominent immune signature in CASP8-MT HNSCs.

Gene enrichment analysis was performed using tools available at the Gene Ontology

Consortium (A), as well as using the Gene Set Enrichment Analysis tool (B and C). A.

Enrichment analysis was performed using genes with FDR $<0.001$ and/or showing log2FC greater than 1.3 or less than -1.3. The top three g ene ontology terms, based on enrichment scores, among the PANTHER GO-Slim Biological Processes significantly enriched in these gene lists are indicated along with Bonferroni-corrected P-values. B. GSEA was performed using a pre-ranked list generated using log2FC values from the edgeR analysis. GSEA Hallmark gene sets enriched in CASP8-MT HNSCs (plots 1 to 5) or CASP8-WT HNSCs (plots 6 to 8) with $F D R<25 \%, P$-value $<0.01$, and showing enrichment at the top or bottom of the list are shown. C. Enrichment plot of a GSEA performed with the pre-ranked list in panel B and a gene set of human orthologs of the genes up regulated in the skin epidermis of Casp- ${ }^{F-K 5}$ Cre mice (CASP8-KOSET) is shown (FDR $<25 \%$, P-value $<0.01$ ). 
A

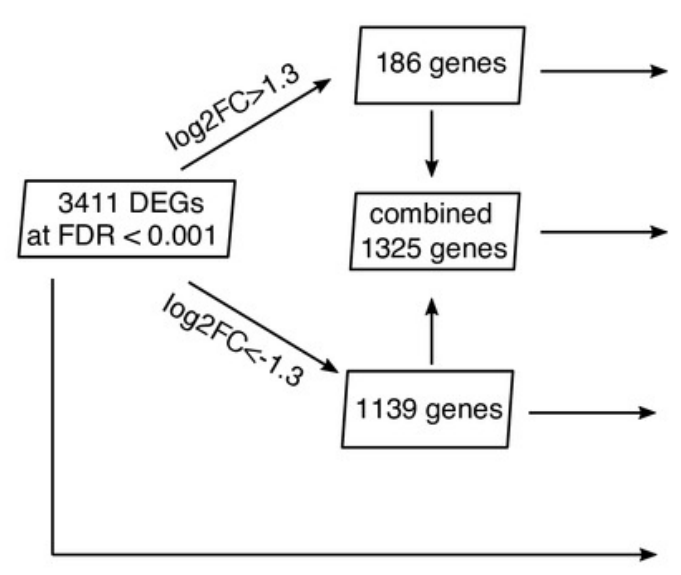

GSEA Enrichment Plots (FDR<25\%, P-value<0.01): CASP8-MT versus CASP8-WT HNSCs using Hallmark gene sets

Enrichment plot: HALLMARK_ALLOGRAFT_REJECTION
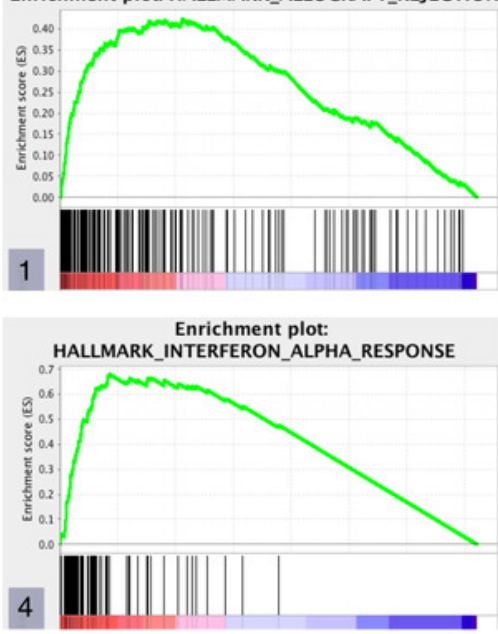

Enrichment plot:
HALLMARK_EPITHELIAL_MESENCHYMAL_TRANSITION

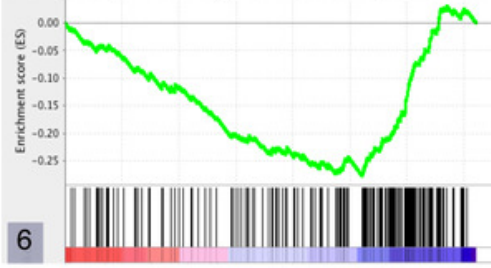

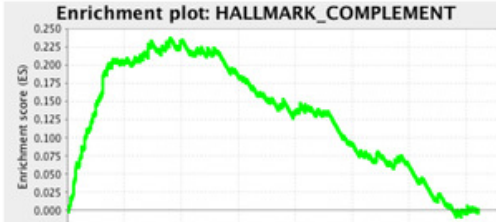

2
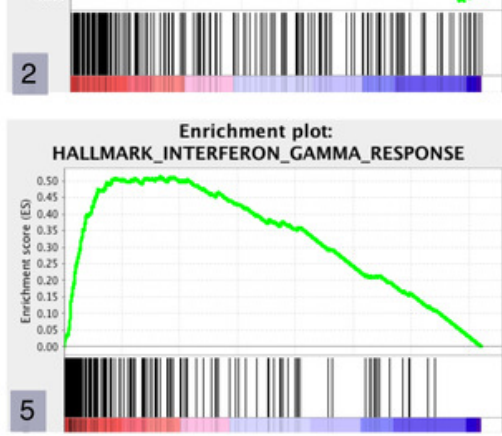

Enrichment plot: HALLMARK_KRAS_SIGNALING_DN

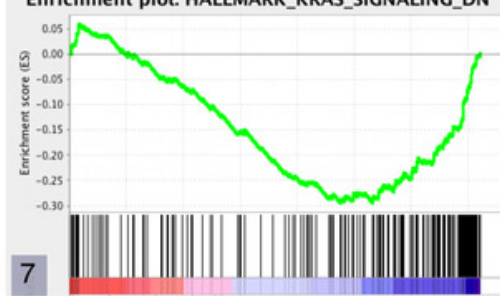

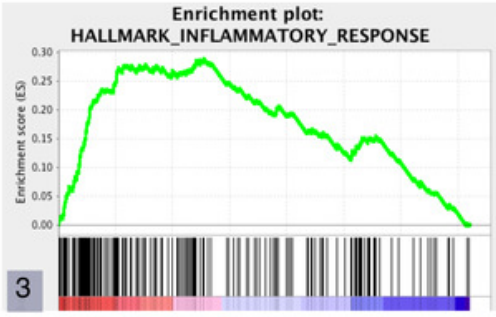

Enrichment plot: HALLMARK_MYOGENESIS

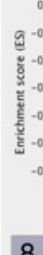

$1.74 \quad 8.11 \mathrm{E}-04$ $6.22 \mathrm{E}-03$

C

GSEA Enrichment Plot: CASP8-MT versus CASP8-WT using CASP8-KOSET (FDR $<25 \%$, P-value $<0.01$ )

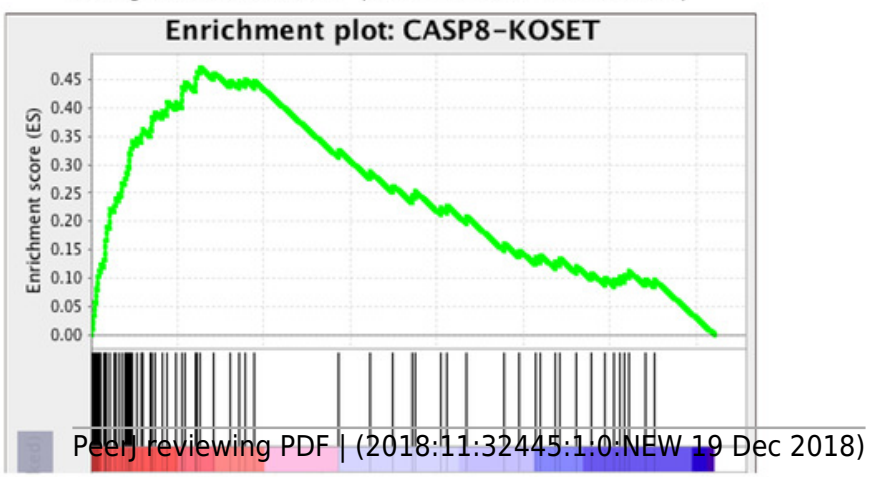




\section{Figure 3}

CASP8-MT HNSCs have higher numbers of certain types of infiltrating immune cells compared to CASP8-WT HNSCs.

Immune cell infiltration levels in CASP8-WT (blue-filled circles), CASP8-MT (red-filled circles) (both HPV-negative), and HPV-positive (black-filled circles) HNSCs were compared using the immune cell infiltration data available at TIMER. Boxplots showing the levels of CD8 ${ }^{+} \mathrm{T}$ cells, neutrophils, and dendritic cells (A), as well as $C D 4^{+} T$ cells and B cells (B) in the three HNSC subsets are displayed. Significance testing was performed using the unpaired two-sided Wilcoxon test. All comparisons with P-value $<0.005$ were considered significant and are indicated in the plots.

A
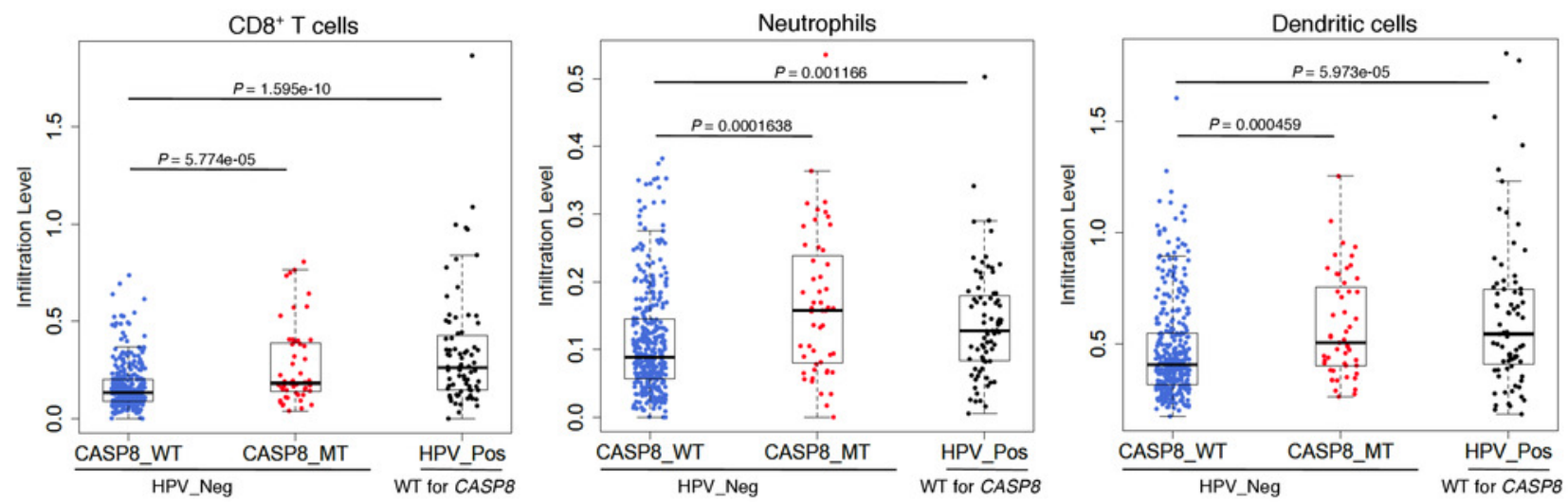

B
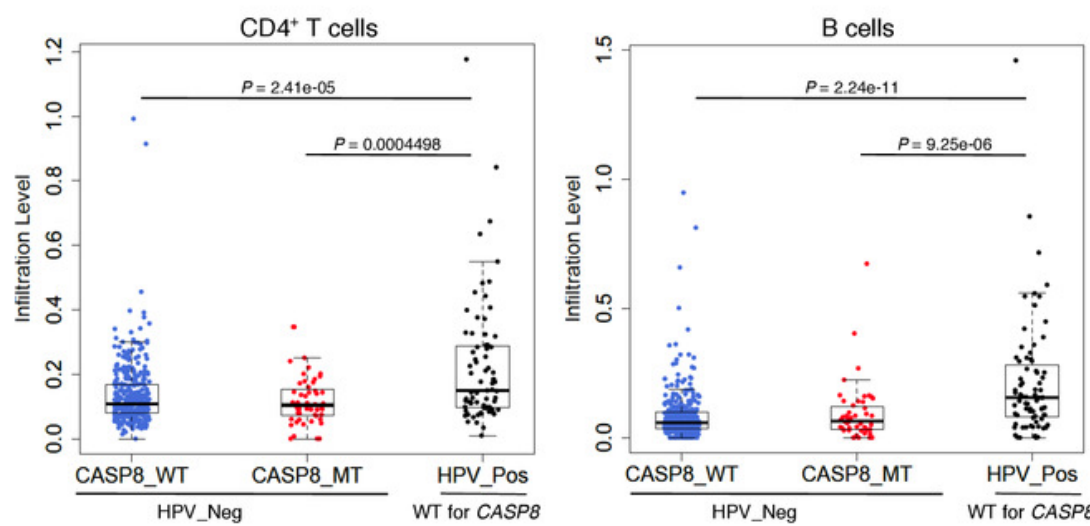


\section{Figure 4}

Survival analysis indicates lack of a survival advantage in CASP8-MT HNSCs in spite of their immune signature.

A. Kaplan-Meier plots showing the survival probability of patients with CASP8-WT or CASP8-MT HNSC tumors (filtered as per the schema in figure 1). Log-rank test was used to compare the two curves and the log-rank P-value is indicated. B. Survival plots generated using the Cutoff Finder tool showing the influence of the expression levels of CD3D and the levels of CD8 ${ }^{+}$T cells on overall survival in CASP8-WT (left) and CASP8-MT (right) cases. Gene expression data was obtained from FPKM-UQ files at TCGA and immune cell infiltration data was obtained from TIMER. 
Figure 4

A

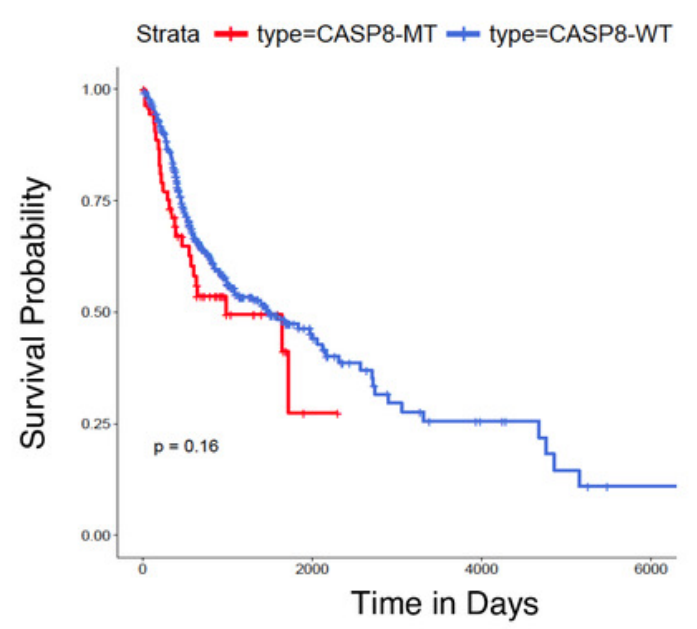

B
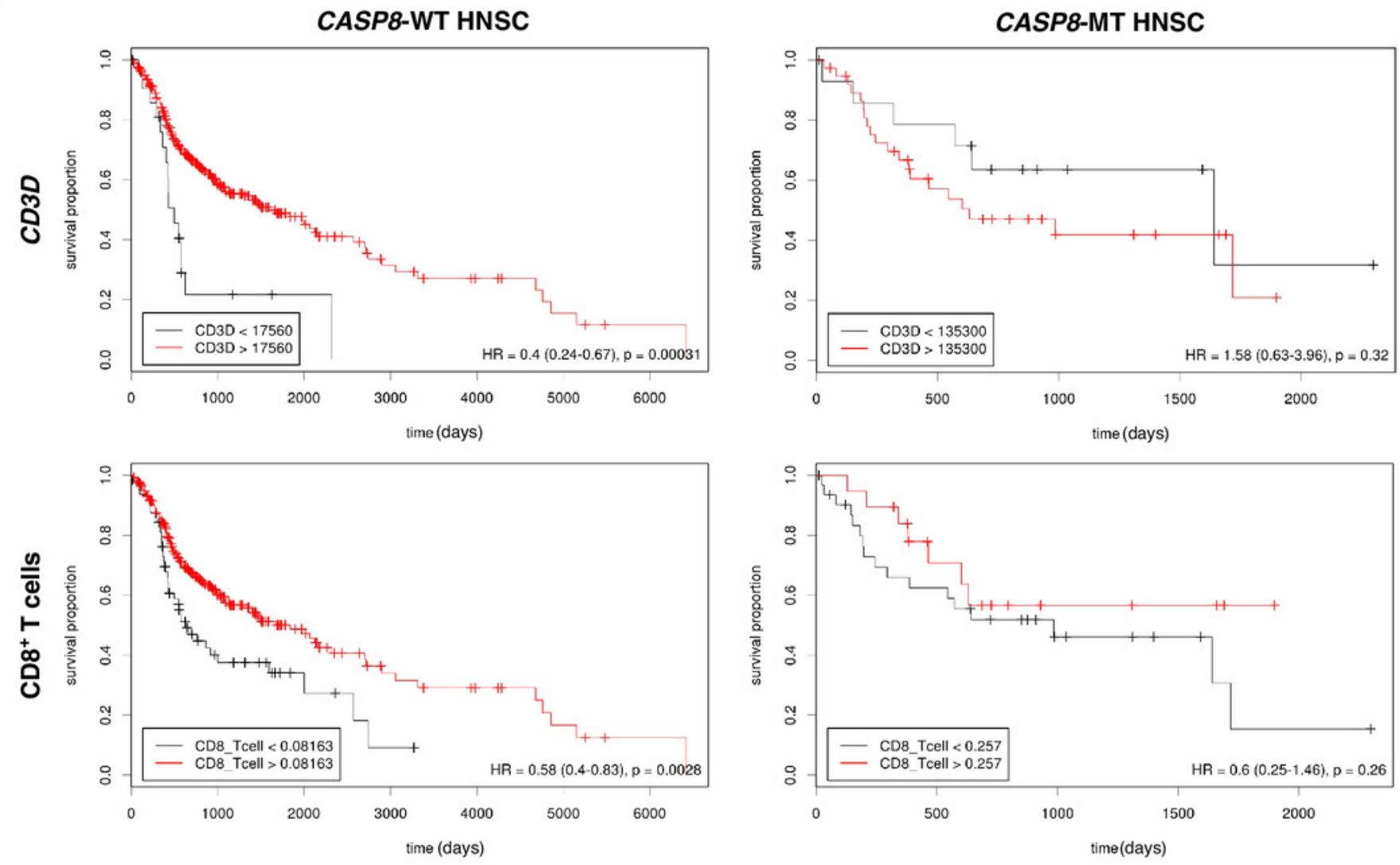


\section{Figure 5}

CASP8-MT UCECs display an immune gene signature, have higher numbers of certain types of infiltrating immune cells, and survive better than CASP8-WT UCECS

A. GSEA was performed using a pre-ranked list generated using log2FC values from the edgeR analysis. Some GSEA Hallmark gene sets enriched in CASP8-MT UCECs (plots 1 to 5) are shown. B. Immune cell infiltration levels in CASP8-WT (blue-filled circles) and CASP8-MT (red-filled circles) UCECs were compared using the immune cell infiltration data available at TIMER. Boxplots showing the levels of CD8 ${ }^{+} \mathrm{T}$ cells and dendritic cells in the two UCEC groups are displayed. Significance testing was performed using the unpaired two-sided Wilcoxon test. All comparisons with P-value $<0.005$ were considered significant and are indicated in the plots. C. Kaplan-Meier plots showing the survival probability of patients with CASP8-WT or CASP8-MT UCEC tumors. Log-rank test was used to compare the two curves and the log-rank $\mathrm{P}$-value is indicated. 
A

GSEA Enrichment Plots: CASP8-MT versus CASP8-WT UCECs using Hallmark gene sets
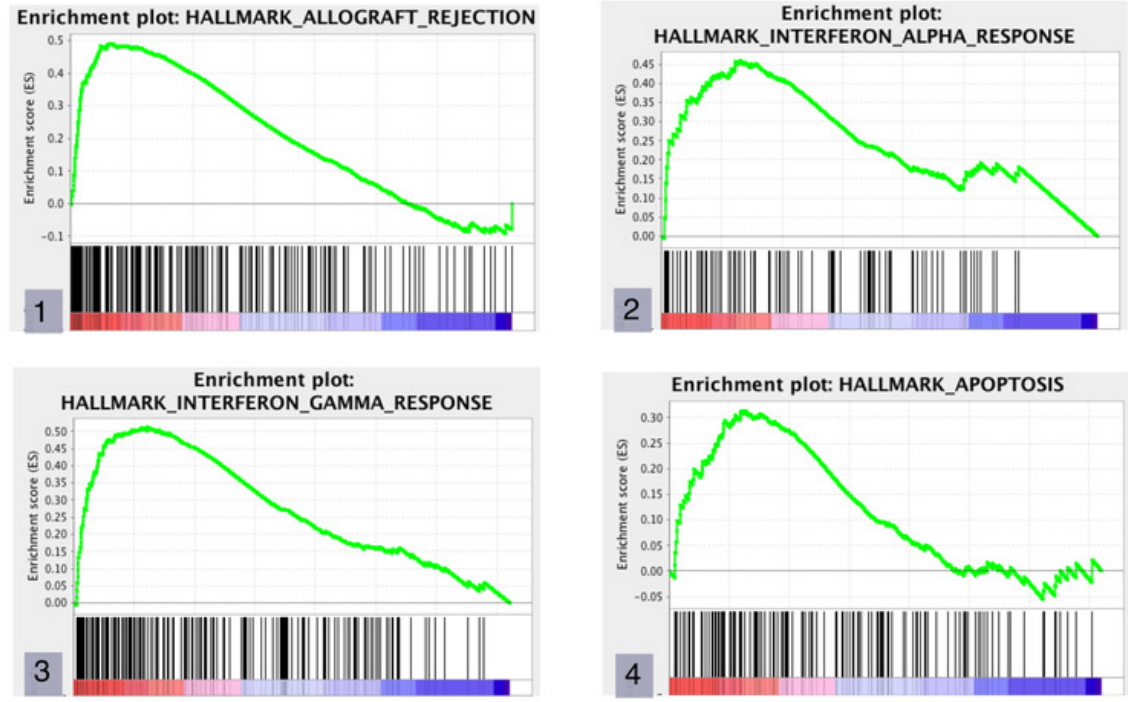

B

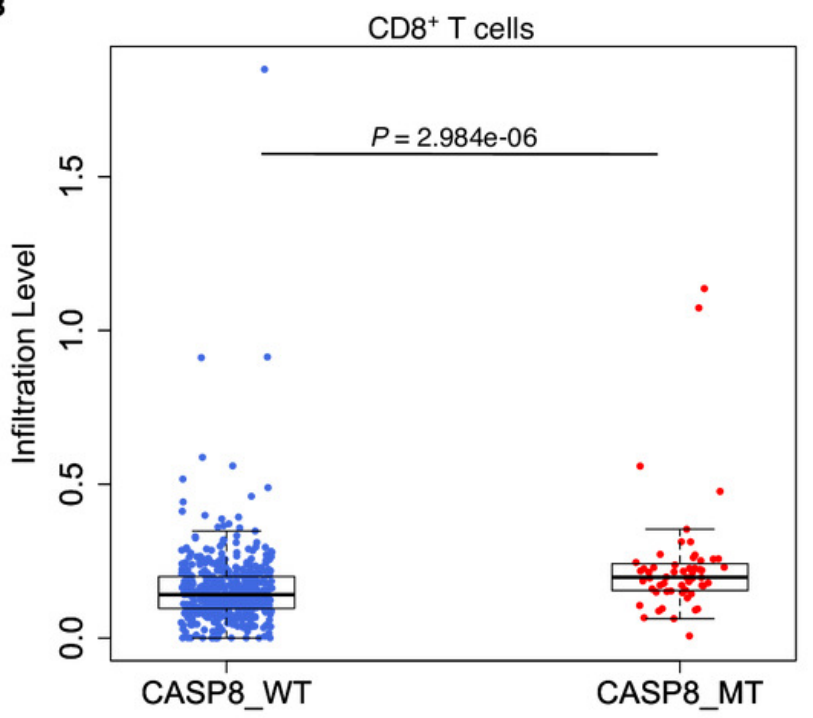

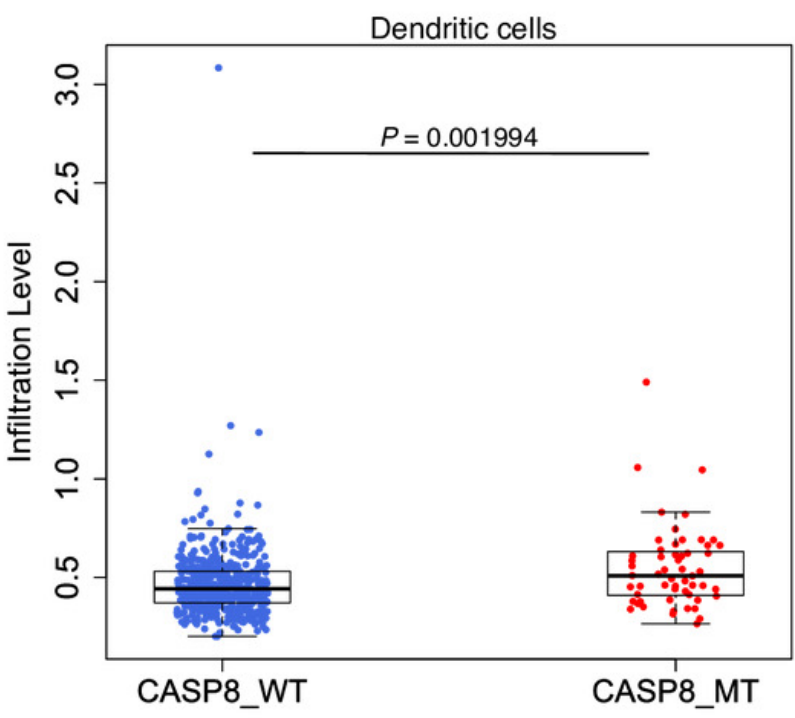

C

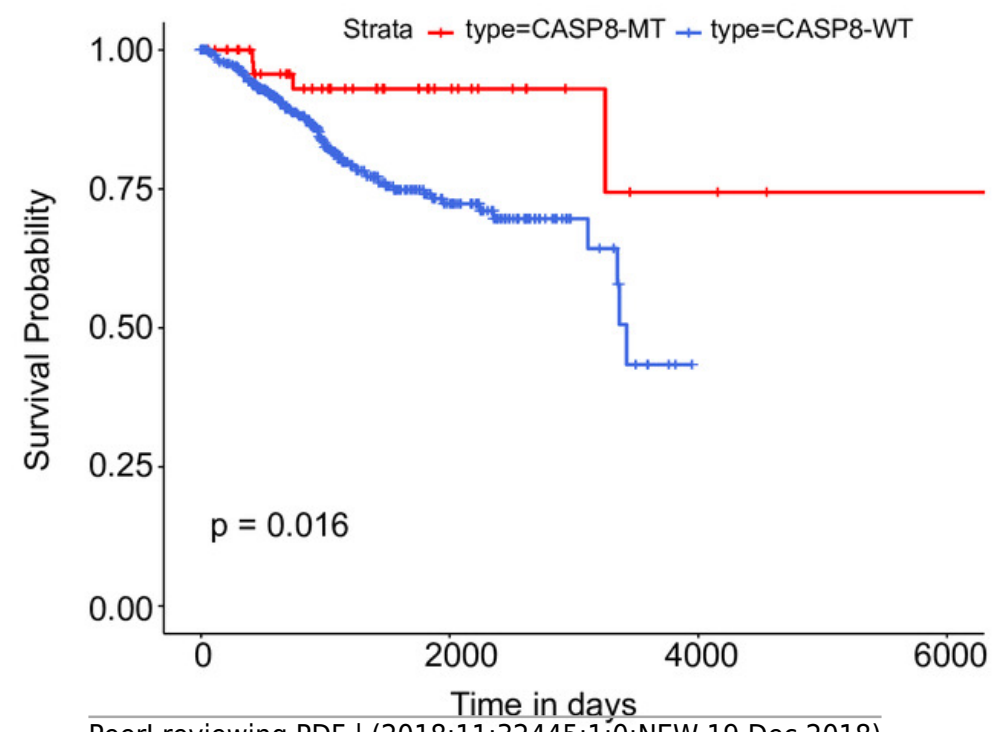

PeerJ reviewing PDF | (2018:11:32445:1:0:NEW 19 Dec 2018) 